\title{
Cycles Embedding in Conditional Faulty Enhanced Hypercubes
}

\author{
R.L. Qin, H.M. Liu \\ College of Science \\ Three Gorges University \\ China
}

\begin{abstract}
Let $f_{e}$ (respectively, $f_{v}$ ) denote the number of faulty links (respectively, nodes) of an $n$-dimensional enhanced hypercube $Q_{n, k}$. In this paper, we showed that $Q_{n, k}(n \geq 5)$ contains a fault-free cycle of length at least $2^{n}-2 f_{v}$, under the constraints that (1) $f_{e}+f_{v} \leq 2 n-3, f_{e} \geq k$ and (2) every node in $Q_{n, k}$ is incident with at least two fault-free links. This result improves the previous known results.
\end{abstract}

Keywords-enhanced hypercube; fault-tolerant embedding; cycle embedding

\section{INTRODUCTION}

In practical systems, the components and communication channel failures can hardly be avoided. So it is practically meaningful to consider faulty networks. The faulty networks has received some researchers attention in the recent years. Let $f_{v}$ and $f_{e}$ be the numbers of faulty nodes and faulty links in $Q_{n, k}$, [1] showed that a fault-free cycle of length at least $2^{n}-2 f_{v}$ can be embedded on $Q_{n, k}$ with $f_{e} \leq n-1$ and $f_{e}+f_{v} \leq 2 n-4$. [2] further showed that a fault-free cycle of length at least $2^{n}-2 f_{v}$ can be embedded on $Q_{n, k}$ with $f_{e}+f_{v} \leq 2 n-4, f_{e} \geq n$ and every node in $Q_{n, k}$ is incident with at least two fault-free links. In this paper, we showed that a fault-free cycle of length at least $2^{n}-2 f_{v}$ can be embedded on $Q_{n, k}(n \geq 5)$ under the constraints that (1) $f_{e}+f_{v} \leq 2 n-3, f_{e} \geq k$ and (2) every node in $Q_{n, k}$ is incident with at least two fault-free links.

\section{PRELIMINARIES}

A graph $G=(V, E)$ is an ordered pair in which $V$ denotes the set of nodes and $E$ denotes the set of links. A path $P\left[v_{0}, v_{m}\right]=\left(v_{0}, v_{1}\right) \oplus\left(v_{1}, v_{2}\right) \cdots \oplus\left(v_{m-1}, v_{m}\right)$ where all the nodes are distinct except that possibly the path is a cycle when $v_{0}=v_{m}$. A node is fault-free if it is not faulty. A link is fault-free if the two end-nodes and the link between them are not faulty. A path (cycle) is fault-free if it contains neither faulty nodes nor faulty links. We call a node $l$ - free if it is exactly incident with $l$ fault-free links.
Definition $2.1 \quad$ Enhanced hypercube $Q_{n, k}=(V, E)(1 \leq k \leq n-1)$ is an undirected simple graph. It has the same nodes of $Q_{n}, V=\left\{x_{1} x_{2} \cdots x_{n}: x_{i}=0\right.$ or $\left.1,1 \leq i \leq n\right\}$. Two nodes $x=x_{1} x_{2} \cdots x_{n}$ and $y$ are connected by an edge of $E$ if and only if $y$ satisfies one of the following two conditions:

(1) $y=x_{1} x_{2} \cdots x_{i-1} \bar{x}_{i} x_{i+1} \cdots x_{n}, 1 \leq i \leq n \quad$ or $y=x_{1} x_{2} \cdots x_{k-1} \bar{x}_{k} \bar{x}_{k+1} \cdots \bar{x}_{n}$.

The enhanced hypercube is the extension of the hypercube by adding the edges $\left(x_{1} x_{2} \cdots x_{n}, x_{1} x_{2} \cdots x_{k-1} \bar{x}_{k} \bar{x}_{k+1} \cdots \bar{x}_{n}\right)$, which called complementary edges of $Q_{n, k}$. Let $E_{c}=\left\{(u, \bar{u}) \in E\left(Q_{n, k}\right) \mid h(u, \bar{u})=n-k+1\right\}$ be the set of all complementary edges. The folded hypercube $F Q_{n}$ is regarded as the special case of the enhanced hypercube when $k=1$. The enhanced hypercube $Q_{n, k}$ can be partitioned into two subgraphs along some component $i(1 \leq i \leq n)$. We use $Q_{n-1, k}^{i 0}\left(Q_{n-1}^{i 0}\right)$ and

$Q_{n-1, k}^{i 1}\left(Q_{n-1}^{i 1}\right)$ to denote the two subgraphs respectively. When $i<k \quad, \quad Q_{n, k}=Q_{n-1, k}^{i 0} \cup Q_{n-1, k}^{i 1} \quad$, when $i \geq k$, $Q_{n, k}=Q_{n-1}^{i 0} \cup Q_{n-1}^{i 1}$.

Lemma 2.1[3] If $n$ and $k$ have different parity, then every edge of $Q_{n, k}$ lies on a cycle of every odd length from $n-k+2$ to $2^{n}-1$.

Lemma 2.2[1] $Q_{n, k}$ contains a fault-free cycle of length at least $2^{n}-2 f_{v}$ if $f_{e} \leq n-1$ and $f_{e}+f_{v} \leq 2 n-4$.

Lemma 2.3[2] $Q_{n, k}$ contains a fault-free cycle of length at least $2^{n}-2 f_{v}$ if (1) $f_{e}+f_{v} \leq 2 n-4, f_{e} \geq n$ and (2)every node in $Q_{n, k}$ is incident with at least two fault-free links.

Lemma 2.4[4] $F Q_{n}(n \geq 4)$ contains a fault-free cycle of length at least $2^{n}-2 f_{v}$ if $f_{v}=2 n-3$.

Lemma 2.5[5] $F Q_{n}$ contains a fault-free cycle of length at least $2^{n}-2 f_{v}$ if (1) $f_{e}+f_{v} \leq 2 n-3, f_{e} \geq 1$ and (2) every node in $F Q_{n}$ is incident with at least two fault-free links. 
Lemma 2.6[6] $Q_{n, k}$ contains a fault-free cycle of length $2^{n}$ if $f_{e} \leq 2 n-3$ and every node in $Q_{n, k}$ is incident with at least two fault-free links.

Lemma 2.7[1] $Q_{n, k}-E_{i}$ is isomorphic to $Q_{n}$ (represented as $Q_{n, k}-E_{i} \cong Q_{n}$ ), where $i \in\{k, k+1, \cdots, n, c\}$.

Lemma 2.8[7] $Q_{n}$ contains a fault-free cycle of length at least $2^{n}-2 f_{v}$ if (1) $f_{e}+f_{v} \leq 2 n-4, f_{e} \leq 2 n-5$ and (2) every node in $Q_{n}$ is incident with at least two fault-free links.

Lemma 2.9[1] $Q_{n, k}$ contains a fault-free path $P[u, v]$ of length $\quad 2^{n}-2 f_{v}-1 \quad$ (respectively $2^{n}-2 f_{v}-2 \quad$ ) if $f_{e}+f_{v} \leq n-1$ when $d_{Q_{n, k}}(u, v)$ is odd (respectively, $d_{Q_{n, k}}(u, v)$ is even).

\section{MAIN RESULTS}

Lemma 3.1 If $f_{e}+f_{v} \leq 2 n-3$, then there exists at most two 2- free nodes in $Q_{n, k}(1 \leq k \leq n-1, n \geq 4)$.

Proof Assume that $Q_{n, k}$ contains three 2- free nodes $u, v$ and $w$. By Lemma 2.1, we know that $Q_{n, k}$ contains a fault-free cycle of length 3 when $k=n-1$. Let us consider a special case that the cycle of length 3 contained by $C_{3}=(u, v) \oplus(v, w) \oplus(w, u)$ and these three links are all faulty links (in this case, we can obtain the least number of faulty elements). The least total number of faulty elements is $(n-1)+(n-2)+(n-3)=3 n-6>2 n-3(n \geq 4)$, which contradicts to the assumption that $f_{e}+f_{v} \leq 2 n-3$. Thus, $Q_{n, k}$ contains at most two 2- free nodes.

Lemma 3.2 If $f_{e}+f_{v} \leq 2 n-3$ and $Q_{n, k}$ contains exactly two 2- free nodes $u$ and $v$, then $d(u, v)=1$ and $u v \in F_{e}$.

Proof If $d(u, v)>1$, then the least total number of faulty links is $(n-1)+(n-1)=2 n-2>2 n-3$. If $u v \notin F_{e}$, then the least total number of faulty links is $(n-1)+(n-1)=2 n-2>2 n-3$. It is contradict to the assumption.

Theorem $3.1 Q_{n, k}-F_{e}-F_{v}$ for $n \geq 5$ contains a fault-free cycle of length at least $2^{n}-2 f_{v}$ if (1) $f_{e}+f_{v} \leq 2 n-3, f_{e} \geq k$ and (2) every node in $Q_{n, k}$ is incident with at least two fault-free links.

Proof Let $F_{v}$ and $F_{e}$ be the set of faulty vertices and faulty edges in $Q_{n, k}$ respectively, $\left|F_{v}\right|=f_{v},\left|F_{e}\right|=f_{e}, F_{e}^{i}=F_{e} \cap E_{i}$ $(i \in\{1,2, \cdots, n, c\})$. If $f_{e}+f_{v} \leq 2 n-4$, by Lemma 2.2 and Lemma2.3, the theorem holds. If $f_{e}+f_{v}=2 n-3$ and $k=1$,

then $Q_{n, 1}=F Q_{n}$, by Lemma 2.4 and Lemma 2.5, the theorem holds. If $f_{v}=0$ and $f_{e}=2 n-3$, by Lemma 2.6, the theorem holds. If $f_{e}+f_{v}=2 n-3, f_{v} \geq 1, f_{e} \geq k$,

we discuss as follows according to the number of 2-free nodes by Lemma 3.1.

Case $1 Q_{n, k}$ contains no 2- free node. We have the following scenarios.

Case $1.1 F_{e}^{k} \cup F_{e}^{k+1} \cdots \cup F_{e}^{n} \cup F_{e}^{c} \neq \phi$. We can choose a link dimension $j$

( $j \in\{k, k+1, \cdots, n, c\})$ such that $f_{e}^{j} \geq 1$. By Lemma 2.7, we have that $Q_{n, k}-E_{j}$ is isomorphic to a hypercube $Q_{n}$ (represented as $Q_{n, k}-E_{j} \cong Q_{n}$ ). Since $f_{v} \geq 1$ and

$$
f_{e}+f_{v}=2 n-3 \text { then } f_{e} \leq 2 n-4 \quad \text { and }
$$
$f_{e}-f_{e}^{j} \leq 2 n-5, f_{v}+f_{e}-f_{e}^{j} \leq 2 n-4$, since every node in $Q_{n, k}-E_{j}$ is incident with at least two fault- free links. We have that $Q_{n, k}-E_{j}$ satisfies the constraints in Lemma 2.8, there exists a fault-free cycle of length $2^{n}-2 f_{v}$ in $Q_{n}-F_{v}-\sum_{i=1}^{n} f_{e}^{i}$, since $Q_{n}-F_{v}-\sum_{i=1}^{n} f_{e}^{i} \subset Q_{n, k}-F_{v}-F_{e}$, we obtain that $Q_{n, k}-F_{v}-F_{e}$ contains a fault-free cycle of length at least $2^{n}-2 f_{v}$.

Case $1.2 F_{e}^{k} \cup F_{e}^{k+1} \cdots \cup F_{e}^{n} \cup F_{e}^{c}=\phi$. That is all the faulty links are in $Q_{n, k}-E_{c}\left(\cong Q_{n}\right)$. We have $i$ - partition on $Q_{n, k}$ to obtain $Q_{n-1, k}^{i 0}$ and $Q_{n-1, k}^{i 1}$ for any $i \in\{1,2, \cdots, k-1\}$, we know that every node in $Q_{n-1, k}^{i 0}$ and $Q_{n-1, k}^{i 1}$ is incident with at least two faultfree links. Let $F_{e}^{i 0}\left(F_{e}^{i 1}\right)$ and $F_{v}^{i 0}\left(F_{v}^{i 1}\right)$ denote the set of faulty links and nodes in $Q_{n-1, k}^{i 0}\left(Q_{n-1, k}^{i 1}\right) .\left|F_{e}^{i 0}\right|=f_{e}^{i 0},\left|F_{e}^{i 1}\right|=f_{e}^{i 1},\left|F_{v}^{i 0}\right|=f_{v}^{i 0},\left|F_{v}^{i 1}\right|=f_{v}^{i 1}$. Let $F_{h}^{i}$ denote the set of $i$-dimensional faulty hypercube links between $Q_{n-1, k}^{i 0}$ and $Q_{n-1, k}^{i 1},\left|F_{h}^{i}\right|=f_{h}^{i}$. Since $f_{e} \geq k \geq 2$ and all the faulty links are distributed in $F_{e}^{1} \cup F_{e}^{2} \cdots \cup F_{e}^{k-1}$, then $\max \left\{\left|F_{e}^{1}\right|,\left|F_{e}^{2}\right|, \cdots,\left|F_{e}^{k-1}\right|\right\} \geq 2$.

Case $\quad$ 1.2.1 $\quad \max \left\{\left|F_{e}^{1}\right|,\left|F_{e}^{2}\right|, \cdots,\left|F_{e}^{k-1}\right|\right\} \geq 3 \quad$, let $f_{h}^{i}=\max \left\{\left|F_{e}^{1}\right|,\left|F_{e}^{2}\right|, \cdots,\left|F_{e}^{k-1}\right|\right\}$, then

$f_{h}^{i} \geq 3 \quad(i \in\{1,2, \cdots, k-1\})$, we can assume that $f_{e}^{i 0}+f_{v}^{i 0} \leq f_{e}^{i 1}+f_{v}^{i 1}$, then

$f_{e}^{i 0}+f_{v}^{i 0} \leq n-3$

( since $\left.2\left(f_{e}^{i 0}+f_{v}^{i 0}\right) \leq f_{e}^{i 0}+f_{v}^{i 0}+f_{e}^{i 1}+f_{v}^{i 1} \leq 2 n-6\right)$ and

$f_{e}^{i 1}+f_{v}^{i 1} \leq 2 n-6$. By lemma 2.2 and lemma 2.3, there exists a fault-free cycle $C_{h}$ of length $2^{n-1}-2 f_{v}^{i 1}$ in $Q_{n-1, k}^{i 1}$, then there are $2^{n-2}-f_{v}^{i 1}$ mutually disjoint links in the 
cycle $C_{h}$ such that each link is different. Since $2^{n-2}>2 n-3$ $(n \geq 5)$ and

$$
\begin{aligned}
& \qquad f_{e}+f_{v}=2 n-3 \\
& \text { then } \\
& 2^{n-2}-f_{v}^{i 1}>f_{e}+f_{v}-f_{v}^{i 1}=f_{e}^{i 0}+f_{v}^{i 0}+f_{e}^{i 1}+f_{h}^{i}>f_{e}^{i 0}+f_{v}^{i 0}+f_{h}^{i} .
\end{aligned}
$$

Consequently, there exists at least one link $(a, b)$ in $C_{h}$ such that $\left(a, a^{i}\right),\left(b, b^{i}\right)$

and $\left(a^{i}, b^{i}\right)$ are all fault-free links. Let $P[a, b]$ be the path between $a$ an $b$ in $C_{h}-\{(a, b)\}$, Whose length is at least $2^{n-1}-2 f_{v}^{i 1}-1$. By Lemma 2.9, a path $P\left[a^{i}, b^{i}\right]$ of length at least $2^{n-1}-2 f_{v}^{i 0}-1$ can be embedded on $Q_{n-1, k}^{i 0}$. Then the

desired fault-free cycle can be constructed as $C=P[a, b] \oplus\left(b, b^{i}\right) \oplus P\left[b^{i}, a^{i}\right] \oplus\left(a^{i}, a\right)$

whose length is least $2^{n-1}-2 f_{v}^{i 0}-1+2+2^{n-1}-2 f_{v}^{i 1}-1=2^{n}-2 f_{v}$.

Case 1.2.2 $\max \left\{\left|F_{e}^{1}\right|,\left|F_{e}^{2}\right|, \cdots,\left|F_{e}^{k-1}\right|\right\}=2$. Without loss of generality, we can assume that $\left|F_{e}^{1}\right| \geq\left|F_{e}^{2}\right| \geq \cdots \geq\left|F_{e}^{k-1}\right|$. Then $\left|F_{e}^{1}\right|=2$, we have 1-partition on $Q_{n, k}$

to obtain $Q_{n-1, k}^{10}$ and $Q_{n-1, k}^{11}$,we can assume that $\quad f_{e}^{10}+f_{v}^{10} \leq f_{e}^{11}+f_{v}^{11} \quad$.Then $f_{e}^{10}+f_{v}^{10} \leq n-3$ and $f_{e}^{11}+f_{v}^{11} \leq 2 n-5$. We have the following cases:

Case 1.2.2.1 $1 \leq f_{e}^{10}+f_{v}^{10} \leq n-3$ and $f_{e}^{11}+f_{v}^{11} \leq 2 n-6$. As Case 1.2.1, we can find a cycle of length $2^{n}-2 f_{v}$ in $Q_{n, k}$.

Case 1.2.2.2 $f_{e}^{10}+f_{v}^{10}=0$ and $f_{e}^{11}+f_{v}^{11}=2 n-5$. Since $f_{v}^{11}=f_{v} \geq 1$, let $u$ be a faulty node in $Q_{n-1, k}^{11}$. By Lemma 2.2 and Lemma 2.3(imagining $u$ not faulty), there exists a cycle $C_{h}$ in $Q_{n-1, k}^{11}$ that does not contain any other faulty elements except $u$ where $h=2^{n-1}-2\left(f_{v}^{11}-1\right)$.

Case 1.2.2.2.1 If $C_{h}$ contains $u$. Let $x$ and $y$ be the two neighbors of $u$ in $C_{h}$. Let $w \neq u$ (respectively, $z \neq u$ ) be a neighbor of $x$ ( respectively, $y$ ) in $C_{h}$.

Case 1.2.2.2.1.1When $\left(x, x^{1}\right)$ and $\left(y, y^{1}\right)$ are two fault-free links in $Q_{n, k}$. Since $Q_{n-1, k}^{10}$ is a fault-free enhanced hypercube, by Lemma 2.9, there exists a path $P_{1}=P\left[x^{1}, y^{1}\right]$ of length $2^{n-1}-2$ in $Q_{n-1, k}^{10}$. Then the fault-free cycle can be constructed as $C=P[y, x] \oplus\left(x, x^{1}\right) \oplus P\left[x^{1}, y^{1}\right] \oplus\left(y^{1}, y\right)$, whose length is at least $2^{n-1}-2+2+2^{n-1}-2\left(f_{v}^{11}-1\right)-2=2^{n}-2 f_{v}$ (see Figure.1).
Case 1.2.2.2.1.2 When $\left(x, x^{1}\right)$ and $\left(z, z^{1}\right)$ are two faultfree links in $Q_{n, k}$ ( the discussion that $\left(y, y^{1}\right)$ and $\left(w, w^{1}\right)$ are two fault-free links is the same). By Lemma 2.9, there exists a path $P_{2}=P\left[x^{1}, z^{1}\right]$ of length $2^{n-1}-1$. Then the fault-free cycle can be constructed as $C=P[z, x] \oplus\left(x, x^{1}\right) \oplus P\left[x^{1}, z^{1}\right] \oplus\left(z^{1}, z\right)$, whose length is at least $2^{n-1}-1+2+2^{n-1}-2\left(f_{v}^{11}-1\right)-3=2^{n}-2 f_{v}$ (see Figure. 2 ).

Case 1.2.2.2.1.3 When $\left(x, x^{1}\right)$ and $\left(y, y^{1}\right)$ are two faulty links in $Q_{n, k}$. Since every node in $Q_{n, k}$ is $l$ - free for some $l \geq 3$. Then the node $x$ is incident to at most $n-4$ faulty elements except $u$ and $\left(x, x^{1}\right)$, and $y$ is incident to at most $n-4$ faulty elements except $u$ and $\left(y, y^{1}\right)$. The most total number of faulty elements that incident to $x$ and the number of faulty elements that incident to $y$ in $Q_{n-1, k}^{11}$ is $n-4+n-4+1=2 n-7<2 n-5$, then there exists a faulty node that does not adjacent to $x$ and $y$ or a faulty edge that its ends are not in $\{x, y\}$. We can assume that there exist a faulty edge $(a, b)$ (the discussion is the same when there exists a faulty node that does not adjacent to $x$ and $y$ ) in $Q_{n-1, k}^{11}$ that its ends are not in $\{x, y\}$. Then by Lemma 2.2 and Lemma 2.3 (imagining $(a, b)$ not faulty), there exists a cycle $C_{1}$ in $Q_{n-1, k}^{11}$ that does not contain any other faulty elements except $(a, b)$ of length $2^{n-1}-2 f_{v}^{11}$. We assume that the cycle $C_{1}$ contains $(a, b)$ (the discussion is easier when $C_{1}$ does not contains $(a, b)$ ), then $\left(a, a^{1}\right)$ and $\left(b, b^{1}\right)$ are two fault-free links. By Lemma 2.9, there exists a path $P\left[a^{1}, b^{1}\right]$ of length $2^{n-1}-1$ in $Q_{n-1, k}^{10}$. Then the desired fault-free cycle can be constructed as $C=P[b, a] \oplus\left(a, a^{1}\right) \oplus P\left[a^{1}, b^{1}\right] \oplus\left(b^{1}, b\right)$, whose length is at least $2^{n-1}-1+2+2^{n-1}-2 f_{v}^{11}-1=2^{n}-2 f_{v}$ (see Figure.3).

Case 1.2.2.2.1.4 When $\left(x, x^{1}\right)$ and $\left(w, w^{1}\right)$ are two faulty edges in $Q_{n, k}$ (the dicussion that $\left(y, y^{1}\right)$ and $\left(z, z^{1}\right)$ are two fault-free links is the same). The most total number of faulty elements that incident to $x$ and the number of faulty elements that incident to $w$ in $Q_{n-1, k}^{11}$ is $n-3+n-4+1=2 n-6<2 n-5$, then there exists a faulty node $h$ that does not adjacent to $x$ and $w$ (or a faulty edge that its ends are not in $\{x, w\}$ ). Let $m$ and $n$ be the two neighbors of $h$. As Case 1.2.2.2.1.1, we can find a cycle $C_{1}$ of length $2^{n}-2 f_{v}$ in $Q_{n, k}$ (see Figure.4)(the discussion is easier when $C_{1}$ does not contain $h$ ). 


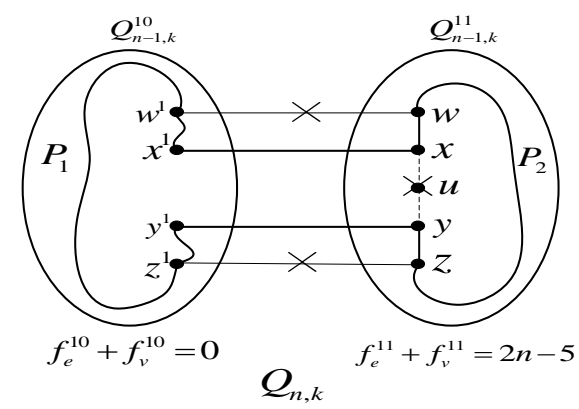

FIGURE I. THE FAULT-FREE CYCLE 1

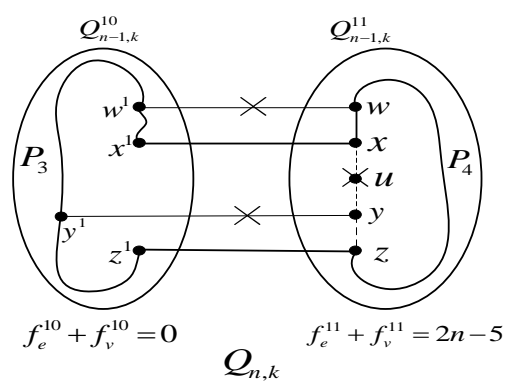

FIGURE II. THE FAULT-FREE CYCLE 2

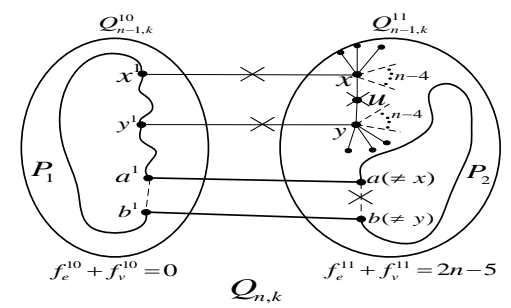

FIGURE III. THE FAULT-FREE CYCLE 3

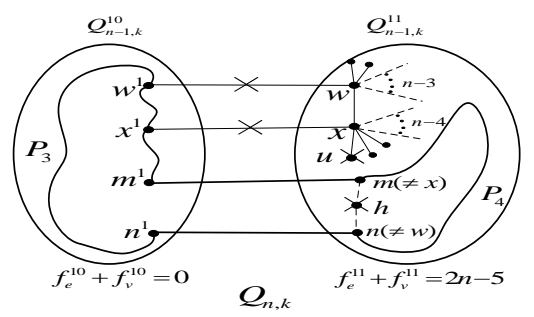

FIGURE IV. THE FAULT-FREE CYCLE 4

Case 1.2.2.2.2 If $C_{h}$ does not contain $u$. As Case 1.2.1, we can find a cycle of length $2^{n}-2 f_{v}$ in $Q_{n, k}$.

Case $2 Q_{n, k}$ contains exactly one 2- free node. There is a unique 2- free node $u$ in $Q_{n, k}$ and every node in $Q_{n, k}-\{u\}$ is $l$ free for some $l \geq 3$. In this case, the number of faulty links incident with $u$ is $n-1$. There exists a dimension $j \in\{k, k+1, \cdots, n, c\}$ such that $u u^{j}$ is a faulty edge (otherwise, let $\left(u, u^{i}\right)$ be the faulty link which is incident with $u$, then $i \in\{1,2, \cdots, k-1\}$, so the number of faulty links incident with $u$ are at most $k-1$, but $k \leq n-1$, therefore, $k-1 \leq n-2$, which is a contradiction), by Lemma 2.7, we have that $Q_{n, k}-E_{j}$ is isomorphic to a hypercube $Q_{n}$. With the same discussion as Case 1.1, we obtain that $Q_{n, k}-F_{v}-F_{e}$ contains a fault-free cycle of length $2^{n}-2 f_{v}$.

Case $3 Q_{n, k}$ contains exactly two 2- free nodes $u$ and $v$. By Lemma 3.2, $d(u, v)=1$ and $u v \in F_{e}$.

Case $3.1(u, v) \in F_{e}^{j}$, and $j \in\{k, k+1, \cdots, n, c\}$, we obtain that $Q_{n, k}-E_{j} \cong Q_{n}$. The same discussion as Case 1.1, we obtain that $Q_{n, k}-F_{v}-F_{e}$ contains a fault-free cycle of length $2^{n}-2 f_{v}$.

Case $3.2(u, v) \in F_{e}^{i}$, and $i \in\{1,2, \cdots, k-1\}$. We can execute an $i$ - partition on $Q_{n, k}$ to obtain $Q_{n-1, k}^{i 0}$ and $Q_{n-1, k}^{i 1}$ such that $u \in Q_{n-1, k}^{0}$ and $v \in Q_{n-1, k}^{i 1}$. Since both $u$ and $v$

are 2- free nodes, the total number of faulty elements are $n-2$ which incident with $u$ in $Q_{n-1, k}^{i 0}$ (the same is $v$ ), then we have that $f_{e}^{i 0}+f_{v}^{i 0} \geq n-2$ and $f_{e}^{i 1}+f_{v}^{i 1} \geq n-2$. Since $f_{e}+f_{v}=2 n-3$ and $\left|F_{e}^{i}\right| \geq 1$, then $f_{e}^{i 0}+f_{v}^{i 0}=n-2$, $f_{e}^{i 1}+f_{v}^{i 1}=n-2$ and $\left|F_{e}^{i}\right|=1$. By Lemma 2.2, Lemma 2.3 and Lemma 2.9 with the same discussion as Case 1.2.1, we can find a cycle of length $2^{n}-2 f_{v}$ in $Q_{n, k}$.

By combining the above cases, we complete the proof.

\section{REFERENCES}

[1] M. Liu, H.M. Liu, Paths and cycles embedding on faulty enhanced hypercube networks. Acta Mathematica Scientia, 33B(1), pp. 227-246, 2013.

[2] R.L. Qin, H.M. Liu, A Further Result on Cycles in Conditional Faulty EnhancedHypercubes. International Journal of Applied Mathematics and Statistics, 52(2), pp.49-54, 2014.

[3] H.M. Liu, The structural features of enhanced hypercube networks. The 5th International Conference on Natural Computation, pp. 345-348, 2009.

[4] Y.H. Fan, H.M. Liu, Path and cycles in faulty folded hypercube. Journal of Mathematics, 33(3), pp. 393-400, 2013.

[5] S.L. Cao, H.M. Liu, On constraint fault-free cycles in folded hypercube. International Journal of Applied Mathematics and Statistics, 42(12), pp. 38-44, 2013.

[6] M. Liu, H.M. Liu, Conditional Edge-Fault-Tolerant Hamiltonicity of Enhanced Hypercube. International Conference on Computer Science and Service System, pp. 297-300, 2011.

[7] Z.Z. Du, J. Jin, M.J. Ma, Cycle embedding in hypercubes with faulty vertices and edges. Journal of University of Science and Technology of China, 38(9), pp.1020-1024, 2008. 